a panel painted with a black and white pattern like that of the rearing pens and a panel painted with yellow and red horizontal stripes 1.5 in. wide. The pattern resembling the rearing pens was used as the negative stimulus. A control group of six chicks was required to discriminate between the yellow and red panel and a grey panel painted with white circles 2.5 in. in diameter and arranged so they formed neither vertical nor horizontal lines. The yellow and red pattern was used as the positive stimulus for three of the control chicks and the grey and white pattern was used as the positive stimulus for the other three. A trial was conducted in the following way. A chick was released from the box in the middle of the runway and, as soon as it had approached to within $1 \mathrm{ft}$. of either panel, the box was lowered again, thus preventing the chick from reaching the other panel. If the chick had approached the positive stimulus it was allowed to feed for $30 \mathrm{sec}$; if it had approached the negative stimulus it was removed from the alley at once.

The experimental chicks, which had the familiar black and white pattern as the negative stimulus, took an average of $55 \cdot 5$ trials before reaching a criterion of 18 correct responses to the positive stimulus in 20 trials. The control chicks, which were required to discriminate between two unfamiliar patterns, took an average of 68.8 trials to reach criterion. The difference between the two groups is statistically significant $(U=5, P=0.021)$. In the first ten trials the experimental group approached the familiar negative stimulus significantly more often than the positive stimulus $(t=3.00, P<0.05)$.

The experiment is incomplete because the results are open to the interpretation that, regardless of their previous experience, the chicks were better able to discriminate between the black and white and the yellow and red patterns than between the yellow and red and the grey and white. Nevertheless, the results suggest that familiarity with the negative stimulus enabled the chicks to learn the discrimination more readily. Further evidence for their familiarity with the negative stimulus was provided by their preference for it in the first ten trials.

Static patterns were used to train the birds, because this simplified the design of the experiment. Moving objects are, of course, conventionally used in imprinting experiments and are certainly more effective than static objects. However, earlier experiments had indicated that static objects are not totally ineffective and can be used to initiate the imprinting process ${ }^{7,8}$.

Even though the evidence is still equivocal, it seems likely that imprinting in chicks not only affects the choice of objects towards which filial and later sexual responses are directed, but also has a more general effect on perceptual development. While the long-term effect of imprinting on sexual preferences is of undeniable functional importance it is probably not a special feature of the learning process.

Sub-Department of Animal Behaviour,

$$
\text { P. P. G. BATESON* }
$$

Madingley, University of Cambridge.

- Present address: Department of Psychiatry (Neuropsychology), Stanford University School of Medicine, California.

${ }^{1}$ Lorenz, K., J. Omithol., 88, 137, 289 (1935).

2 Guiton, P., Anim. Behav., 9, 167 (1961).

s Bambridge, R., Science, 136, 259 (1962).

- Schutz, F., Naturwiss., 50, 624 (1963).

${ }^{5}$ Lawrence, D. H., J. Exp. Psychol., 39, 770 (1949).

- Gibson, E. J., and Walk, R. D., J. Comp. Physiol. Psychol., 49, 239 (1956)

? Bateson, P. P. G., J. Comp. Physiol. Psychol., 57, 100 (1964).

Bateson, P. P. G., J. Comp. Physiol. Psychol. (in the press).

\section{Repetition and Task Difficulty}

A RECENT investigation into the effect of repetition of items on paired associate learning ${ }^{1}$ has indicated that a single repetition of a presented item aids learning but that further presentations of the item yield no additional benefit. The design of this experiment involved the replacement of all items not correctly associated on a trial with randomly selected new items. In this way the list length remained constant (12 paired associate letternumber items) and all items on each trial had yet to be learned.

Measurement was made of the probability of correct recall (' $p(C)$ ') after each number of presentations. This index was of the relative frequency type, being the proportion of those items receiving a given number of presentations which are correctly associated. It is convenient to refer to the 'probability of correct recall after one presentation' as $p\left(C_{1}\right)$, 'probability of correct recall after two presentations' as $p\left(C_{2}\right)$, and so on. The results referred to above may now be described in these terms: $p\left(C_{2+}\right)$ was found to be significantly greater than $p\left(C_{1}\right)$, but $p(C)$ did not increase beyond $p\left(C_{2}\right)$.

It is felt that the conditions of this experiment constituted a relatively difficult task: time-intervals were short, and subjects were required to repeat aloud items during each presentation. Two experiments are reported here in which the same design was used but in which task difficulty was systematically varied.

In the first of these speed of presentation was decreased. Presentation time was now 3 sec followed by a 5 -sec inter-item interval, and subjects were not required to repeat aloud items as they were exposed. All othor variables, such as procedure, materials, subject population, instructions, etc., were the same as previously. In these less difficult conditions it was found that $p\left(\ddot{C}_{2+}\right)$ was not significantly different from $p\left(C_{1}\right)$.

The level of task difficulty is also partly determined by conditions during recall tests. A further experiment was, therefore, conducted in which presentation conditions were those of the original experiment, but in which the recall interval available for each item was increased from $5 \mathrm{sec}$ to 10 sec. All other variables were held constant. It was again found that $p\left(C_{2+}\right)$ was not significantly different from $p\left(C_{1}\right)$.

It is therefore concluded that task difficulty is an important variable mediating the effect of repetition on learning. In relatively easy conditions learning may be seen to occur on an all-or-none basis, but when the level of task difficulty is raised an incremental factor becomes operative. The findings from this series of experiments also indicate that relatively minor changes in experimental conditions can significantly affect the consequences of repeated presentation of items. It appears that 'learning' is neither incremental nor all-or-none; rather that learning in some conditions is incremental and that learning in other conditions takes place on an all-or-none basis.

Peter B. WARr

Department of Psychology,

University of Sheffield.

${ }^{1}$ Warr, P. B., Quart. J. Exp. Psychol., 15, 4 (1963).

\section{Effects of Light and Dark on the Acetylcholinesterase Activity of the Retina}

THIs article reports one of a serios of studies designed to elucidate whether acetylcholinesterase (AchE) synthesis is regulated by its substrate, to determine the quantitative parameters of this relationship and to establish whether these relations are functionally significant in neural transmission.

Substrate induced enzyme synthesis in the mammalian organism has been described for several enzymes ${ }^{1}$. In general the inducing substrate has been applied by injection and often in non-physiological doses. For example, studies on the induction of tryptophan pyrrolase require that large doses of tryptophan be given by injection and charactoristically the animal shows signs of sub-lethal toxicity. 